\title{
Performances Management When Modelling Internal Structure of a Production Process
}

\author{
Claudio Pinto ${ }^{1}$ \\ ${ }^{1}$ DISES,University of Salerno, Via Giovanni Paolo II, Fisciano (SA), Italy \\ Correspondence: Claudio Pinto, DISES,University of Salerno, Via Giovanni Paolo II, 132, 84048 Fisciano (SA), \\ Italy. E-mail: clpinto@unisa.it
}

Received: May 5, 2020

doi:10.5539/ijbm.v15n7p133
Accepted: June 2, 2020

Online Published: June 8, 2020

\begin{abstract}
Performance management is a central point for both public and private organizations. In the data envelopment analysis (DEA) method, performance management takes the form of measuring relative efficiency. Furthermore, considering each organization and or production process as a black box, inputs are transformed into outputs. In reality, production organizations or processes are composed of different parts that carry out different related activities. For this reason, modeling the internal structure of a production process like a system of interconnected parts makes it possible to measure its performance at the sub-process level. In this paper, we hypothesized a production process, made up of three interconnected parts. It is a new strategy to acquire relative efficiency consisting of building a block inside the system with at least two sub-processes. This step refers to a basic model of relational Network Data Envelopment Analysis (NDEA). Also, we used the additive decomposition formula to measure the efficiency of the whole process. We highlighted the differences in the measurement, between the direct application of the relational NDEA model and the measurement with the block approach model.We compared the cumulative empirical distribution functions of the efficiency scores of a sub-process with the decomposition formula multiplicative and our approach. In conclusion, the paper proposes, a new strategy to measure the relative performances of a production process model as a network system of three subprocesses, which combines the NDEA and the DEA. This allows us to reevaluate, the indications of policy at the individual sub-process level (block). Moreover, it is a versatile approach which allows aggregation of the sub-processes in blocks, according to the particular policy requirements, legislative technological constraints, etc.
\end{abstract}

Keywords: network data envelopment analysis, modelling internal structure, performance management, private and public organizations

\section{Introduction}

The measurement as the response at real problems solution is the core of the Operation Research (OR) among others (Hiller, et al., 2001). The Data Envelopment Analysis (DEA) (Cooper, et al., 2007) within the management science (MS) and operations research (OR) tradition, occupy an important place as a method for shaping production process and measuring different concepts of efficiency (Note 1)(i.e. technical efficiency, scale efficiency, scope efficiency and so on). The two aspects so far outlined, modelling and measurement,are two fundamental steps in the organizational performances management. Economists and/or operation researchers used mainly two different approaches to economically modeling the production process and measure its efficiency: 1) the econometric approach and 2) the mathematical approach. Inside the first approach, the preferences of the economists fall on the Stochastic Frontier Approach (SFA) (Aigner, et al., 1977); (Meeusen, et al., 1977), Correctly Ordinary Least Square (COLS), Modified Ordinary Least Square (MOLS) (Robinson, 2008) and Maximum Likelihood Estimation (MLE) (Greene, 1980). Inside the operations researcher group the most used approaches are the DEA (Cooper, et al., 2007) and the Free Disposable Hull (FDH) (Deprins, et al., 1984). The main difference between the two groups of scholars is that for those that uses DEA and FDH it is more easy to carry out multi-output production process, meanwhile in the case of the econometric approach it is possible consider error terms (although in the DEA approach much is doing done) (Olesen, et al., 2016). Inside the DEA approach, some author noted that the DEA approach have some weakness that can misleading the efficiency measurement[among others (Daraio, et al., 2008). In particular some of they noted that the DEA do not allow to see inside the production process, modeling it as a "black box" that transform inputs in the outputs (Note 2). Different authors, following this observation developed different approaches/models to modeling the internal 
structure of the DMU inside the DEA approach as well as proposed several formulas/approach to measure its efficiency in the case of basic model with two stages as well as in the case of more than two stages among others (Fa"re, et al., 1995); (Fa"re, et al., 1996a); (Fa"re, et al., 1996b); (Kao, et al., 2008); (Liang, et al., 2006); (Liang, et al., 2008) with different extensions among others (Chen, et al., 2010 b); (Premachandra, et al., 2012), (Castelli, et al., 2004). Inside this literature the internal structure in the case of health care services it has been also considered [among others (Chilingerian, et al., 2004) as well as in others sectors i.e. (Kao, et al., 2008) apply NDEA in non-life insurance companies, (Chen, et al., 2004) in the bank branch, (Sexton, et al., 2003) at major league baseball and so on. The objective of this paper is to modeling the internal structure of a production process with three interdependent subprocesses. At this end, we take as a cue the relational Network Data Envelopment Analysis (NDEA) model proposed in (Pinto, 2016) where the author modeling and measure the relative efficiency of the hospital acute care production process. In particular, here, differently to (Pinto, 2016) are proposing a relational model with tree stages corresponding three different activity taken in the acute care in the hospital setting. In particular we are assuming that the hospital acute care provides the medical activity, the rehabilitative activity, and the assistance activity. The consideration of a third activity (rehabilitative) relatively at the two (medical and assistance) considered in (Pinto, 2016) need to reconsider the relationship among these three activities, as well as will produce a different functioning of the process of the hospital care of the acute. This allow us to build a more general NDEA model to apply in others sectors/activities. Once obtained the relational NDEA model we propose a novel strategy consisting in building a block of two subprocess to estimate the efficiency of the its parties, The paper is structured as follow: in the section 2 we modeling graphically the internal structure of a production process with three stages (three subprocess), and describe its functioning (subsection 2.1). In the section 3 we formulate our relational NDEA model (subsection 3.1) and the way to calculate the relative efficiency of its subprocess (subsection 3.2), in the section 4 we apply the relational NDEA model expanding the model in (Pinto, 2016). Finally in the section 5 we show discussion and conclusions.

\section{Material Studied}

To consider the internal structure of a production process/organization inside DEA context is relatively recent. The classification in (Castelli, et al., 2010) define the main research in this areas as shared flow, multilevel and network models, depending on the assumptions made. For shared flow models the subunits of the DMU have shared inputs and shared outputs. In the multilevel models DMU inputs (outputs) are not necessarily inputs (outputs) of its subunits. In network models the subunits have at least one output which is an input of another different subunits. (Kao, 2014) from measurement point of view classify nine types of models used to measure efficiency of network production process: 1) independent models, 2) system distance measure model, 3) process distance measure model, 4) factor distance measure model 5) slacks-based measure model,6) ratio-form system efficiency model, 7) ratio-form process efficiency model, 8) game theoretic model, 9) value-based model. The independent model treat each part process as independent DMUs and measure their efficiencies separately. The system distance measure model specify a model to find either the minimum input distance measure or maximum output distance measure for the system efficiency. In the system distance measure model every process is required to have the same distance parameter when measuring the system efficiency. An extension of this model is to allow each process to be associated with a different parameter, $\theta^{k}$, which represents the efficiency of each $\mathrm{k}$ process, and the system efficiency is a weighted average of these individual process efficiencies. The factor distance measure allow every factor to have different parameters, and the objective function is to minimize the weighted average of these. Slacks-based measure model measure the system and process efficiencies of a network system. Ratio-form system efficiency model is those proposed by (Kao, 2009(a)) where for general network systems, which requires the same factor to have the same multiplier in the aggregation, no matter which process the factor corresponds to. In contrast to the system efficiency model, where system efficiency is the primary concern in searching for the most favorable multipliers, and a relationship between the system and process efficiencies is then derived, the primary concern of a process efficiency model is process efficiencies, with a driving force of their aggregation. The value-based model is concerned with maximizing either the aggregate output, or the profit, of a network system within the constraints. By structure point of view (Kao, 2014) classify the network system by: 1) basic two-stage structure, 2) general two-stage structure, 3) series structure, 4) parallel structure, 5) mixed structure. 6) dynamic structure, 7) hierarchical structure. In this paper we propose a network system of three subprocesses differently connected, so following (Kao, 2014) our model can fall in to mixed structure and by measurement point of view in to ratio form process efficiency. Following (Castelli, et al., 2010) our network process can be classified as a shared variables models. The distinctive point of the work is that to measure the relative efficiency of a mixed structure we do not apply directly one of the measurement method classify above in (Kao, 2014) but we suggest as first thing to individuated blocks inside it in a way to reduce our model at more simple series or parallel structure and then adopt the some type of measurement, i.e. 
the ratio form, of the new structure. In our knowledge this solution is new.

\section{Modeling a Production Process Considering Its Internal Structure with Three Stages}

In this section, we propose a relational model to describe the hypothetical functioning of a production process with three stages differently connected as those in Figure 1. Our NDEA model formulation will be showed in the following section 4 as well as a we propose a new efficiency measurement strategy (Pinto, 2018).

\subsection{The Description of the Functioning of the Process according to Our Relational Model}

We are modeling a production process as a network system with three sub-process differently connected among them (see Figure 1) and we are considering additional variable as a "common" variable (Castelli, et al., 2010) that provided services all the stages without the possibility to be "shared" among them (i.e. the administrative staff)(Note 3).

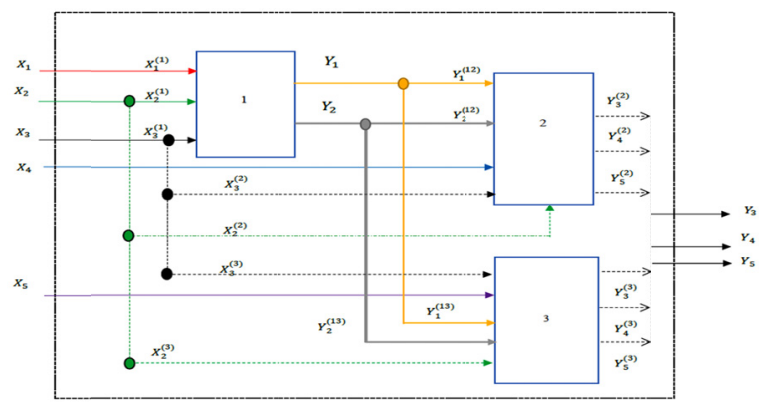

Figure 1. System with three stages

To explain the functionality of the process represented in Figure 1, we refer to the example of hospital acute care services reported in (Pinto, 2016). According to the model in Figure 1, the production process of hospital treatment for acute patients considers three different activities: medical activity (developed with subprocess 1), rehabilitation activity (developed with subprocess 3 ) and assistance (developed with subprocess 2) (Note 4). Rehabilitation services are provided both to surgical patients after surgery (yellow path, Y1) and possibly during the days of hospitalization (gray path, Y2) after patients have or are receiving other medical treatment (stage 1 output) (Note 5). All 3 phases are connected to each other through the use of hospital beds (X2) available for the acute care process (green path) but each phase differs in the personnel involved. In fact, the first phase (phase 1 in Figure 1) or the medical activity, is mainly conducted by the technical-health care staff $\left(X_{3}^{(1)}\right)$ and doctors $\left(X_{1}^{(1)}\right)$. The assistance activity (the second phase, phase 2 in Figure 1) requires technical-health care $\operatorname{staff}\left(X_{3}^{(2)}\right)$ and nurses $\left(X_{4}\right)$. The rehabilitation activity is carried out mainly by the rehabilitation personnel $\left(X_{5}\right)$ and from medical-technical staff $\left(X_{2}^{(3)}\right)$ and receives, as on the other hand happens for the assistance services $\left(Y_{1}^{12}, Y_{2}^{12}\right)$, a part of the outputs of the first phase $\left(Y_{1}^{(13)}, Y_{2}^{(13)}\right)$. The technical-sanitary staff becomes, like the beds read $\left(X_{2}^{(1)} ; X_{2}^{(2)} X_{2}^{(3)}\right)$ a shared variable thus, consolidating the interdependencies between the 3 subprocesses together with the variable bed places. The administrative staff (which however is not included in the model) offer support to the process, for example in the admission phase (before the start of the whole process) and in the discharge phase (after the end of the process). The surgical interventions $\left(Y_{1}\right)$ and the days of hospitalization $\left(Y_{2}\right)$ are the outputs of the first subprocess. At the same time, are the inputs for the other two subprocesses, 2 and 3, as intermediate variables. This means that the central subprocess of this treatment process is subprocess 1 . If no medical activity is carried out, the process does not start. Both the assistance and the rehabilitation activities end when the patients are discharged $\left(Y_{3}, Y_{4}, Y_{5}\right)$ (Note 6).

\section{The Efficiency Measurement}

Inside the OR and MS approach the DEA it has been extensively used to modeling the production process [i.e. (Zhu, 2009); (Ozcan, 2008)] and measuring the performances of different type of Decision Making Units (DMU) [for a review of DEA applications see (Seiford, 1996); (Cooper, et al., 2007); (Cook, et al., 2009); (Liu, et al., 2013)]. However, in the DEA approach the internal structure of a DMU is not considered. To consider the internal structure of a DMU we can consider advanced DEA models [see (Castelli, et al., 2010); (Lewis, et al., 2004); (Kao, 2009(a)); (Kao, 2014)]. In this paper, according to the classification in (Castelli, et al., 2010), we developed a combination between a shared model with a network model (see Figure 1). In the following two sub-sections we will show the NDEA formulation (subsection 4.1) and propose a novel strategy to measure the system efficiency (sub-section 4.2) using a "blocks" to transform the system. 


\subsection{The DEA Model}

Charnes, et al., (1978) proposed a technique to aggregating the outputs in a virtual outputs and the inputs in a virtual inputs and use the ratio virtual output/virtual input to represent the relative efficiency of a production units (named Decision Making Units- DMU). In its dual formulation the technique envelop the observations by production function. The technique is the Data Envelopment Analysis (DEA). Since the functional form need not be specified it is a non parametric approach. So, to measure the performance applying the multiplier formulation of the model we get the following linearized input program (after Charnes transformation):

$$
\begin{gathered}
\max \sum_{r=1}^{s} u_{r} Y_{r o} \text { s.t. } \sum_{\substack{i=1 \\
\sum_{r=1}^{s}}}^{m} v_{i} X_{i o}=1, \\
u, v \geq 0
\end{gathered}
$$

Once solved the program (1) the optimal solution $u^{*}, v^{*}$ are obtained. So, the efficiency will be:

$$
\sum_{r=1}^{s} u_{r}^{*} Y_{r o}
$$

The model above is a DEA "black box" model which is graphically showed in the Figure 2 below.

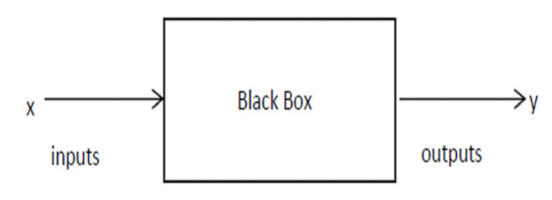

Figure 2. DEA black box

In the following subsection we will show the NDEA models and two techniques to conduct the relative efficiency measurement for the systems (organization/production process) and its parts (divisions/stages) displayed in the Figure 1 above.

\subsection{The NDEA Models Formulation}

In this section we formulate a relational NDEA model to calculate the efficiency of the process in Figure 1. The efficiency measurement of its subprocesss is in the subsection (4.3) where we will use two ways to do this, one of this, according our knowledge, is new and never adopted way. Assuming Constant Return to Scale (CRS) the corresponding input oriented multiplier relational NDEA model for the process displayed in the Figure 1 above[ (Kao, 2017)] (Note 7) will be:

$$
\begin{gathered}
v_{1} Y_{3}+v_{2} Y_{4}+v_{3} Y_{5} \\
\text { s.t. } \quad u_{1} X_{1}+u_{2} X_{2}+u_{3} X_{3}+u_{4} X_{4}+u_{5} X_{5}=1 \\
\left.\left(w_{1} Y_{1}+w_{2} Y_{2}\right)-\left(u_{1} X_{1}^{1}+u_{2}\left(1-\alpha_{1}-\beta_{1}\right) X_{2}^{1}+u_{3}\left(1-\alpha_{2}-\beta_{2}\right) X_{3}^{1}\right) \leq 0 \quad \text { I sub } \quad \text { (model } 1\right) \\
\left(v_{1} \delta_{1} Y_{3}^{(2)}+v_{2} \delta_{2} Y_{4}^{(2)}+v_{3} \delta_{3} Y_{5}^{(2)}\right)-\left(w_{1}\left(1-\gamma_{1}\right) Y_{1}^{(12)}+w_{2}\left(1-\gamma_{2}\right) Y_{2}^{(12)}+u_{4} X_{4}+u_{2} \alpha_{1} X_{2}^{(2)}+u_{3} \alpha_{2} X_{3}^{(2)}\right) \\
\leq 0 I I s u b \\
\left(v_{1}\left(1-\delta_{1}\right) Y_{3}^{(3)}+v_{2}\left(1-\delta_{2}\right) Y_{4}^{(3)}+v_{3}\left(1-\delta_{3}\right) Y_{5}^{(3)}\right) \\
-\left(u_{2} \beta_{1} X_{2}^{(3)}+u_{3} \beta_{2} X_{3}^{(3)}+u_{5} X_{5}+w_{1} \pi_{1} Y_{1}^{(13)}+w_{2} \pi_{2} Y_{2}^{(13)}\right) \leq 0 \text { III sub } \\
\left(v_{1} Y_{1}+v_{2} Y_{2}+v_{3} Y_{3}\right)-\left(u_{1} X_{1}+u_{2} X_{2}+u_{3} X_{3}+u_{4} X_{4}+u_{5} X_{5}\right) \leq 0 \text { system }
\end{gathered}
$$

Where:

$\mathrm{X}_{1}, \mathrm{X}_{2}, \mathrm{X}_{3}, \mathrm{X}_{4}, \mathrm{X}_{5}=$ system resources

$\mathrm{Y}_{3}, \mathrm{Y}_{4}, \mathrm{Y}_{5}=$ outputs system

$\mathrm{X}_{1}^{(1)}, \mathrm{X}_{2}^{(1)}, \mathrm{X}_{3}^{(1)}=$ resources I sub-process

$\mathrm{Y}_{1}, \mathrm{Y}_{2}=$ outputs I sub- process

$\mathrm{Y}_{1}{ }^{(12)}, \mathrm{Y}_{2}{ }^{(12)}=$ relational resources between the I and the II sub-process

$\mathrm{Y}_{1}{ }^{(13)}, \mathrm{Y}_{2}{ }^{(13)}=$ relational resources between the I and the III sub-process

$\mathrm{X}_{2}{ }^{(2)}, \mathrm{X}_{3}{ }^{(2)}=$ shared inputs resources between the I and the II sub-process 
$\mathrm{X}_{4}=$ exogenous resources II sub-process

$\mathrm{Y}_{3}^{(2)}, \mathrm{Y}_{4}{ }^{(2)}, \mathrm{Y}_{5}^{(2)}=$ outputs II sub-process

$\mathrm{X}_{5}=$ resources III sub-process

$\mathrm{X}_{2}^{(3)}, \mathrm{X}_{3}{ }^{(3)}$, = shared resources between I and III sub-process

$\mathrm{Y}_{1}{ }^{(13)}, \mathrm{Y}_{2}{ }^{(13)}=$ shared outputs resources between I and III sub-process

$\mathrm{Y}_{3}{ }^{(3)}, \mathrm{Y}_{4}{ }^{(3)}, \mathrm{Y}_{5}{ }^{(3)}=$ outputs third sub-process

$$
\begin{aligned}
& \alpha_{1}, \alpha_{2}=\text { proportion of the shared inputs variables } X_{2}^{2}, X_{3}^{2} \text { respectively } \\
& \beta_{1}, \beta_{2}=\text { proportion of the shared inputs variables } X_{2}^{3}, X_{3}^{3} \text { respectively }
\end{aligned}
$$

$\gamma_{1}, \gamma_{2}=$ proportion of the shared intermediate variables $Y_{1}^{(12)}, Y_{2}^{(12)}$ respectively and

$\pi_{1}, \pi_{2}=$

proportion of shared intermediate variables between the I and III subprocess $Y_{1}^{(13)}, Y_{2}^{(13)}$ respectively $\delta_{1}, \delta_{2}, \delta_{3}=$ proportion of the shared outputs variables $Y_{3}^{(2)}, Y_{4}^{(2)}$,

$Y_{5}^{(2)}$ and $(1-\delta)$ the proportion of $, Y_{3}^{(3)}, Y_{4}^{(3)}, Y_{5}^{(3)}$

With this formulation the same inputs and outputs will receive the same weights [ (Kao, 2009(a))]. The operation of each process is described with the constraints in the model 1. For example the constraints sub 1 write down the operations of the first subprocesses. The constraint sub 2 consider the operations of the second subprocesses, and so on. The relational nature of the model 1 is that the outputs of the first subprocesses $\left(Y_{1}, Y_{2}\right)$ receive the same weights $\left(w_{1}, w_{2}\right)$ of the relational variables that connect it with the second subprocesses $\left(Y_{1}^{(12)}, Y_{2}^{(12)}\right)$ and third subprocess $\left(Y_{1}^{(13)}, Y_{2}^{(13)}\right)$. So, as stated in others parts in the paper the same variables (in this case $Y_{1}$ and $Y_{2}$ ) receive the same weights. The proportion assigned to the variables of each subprocesses is differently defined (Kao, 2017). Here we assigned a fixed proportion (i.e. $\alpha_{1}, \alpha_{2}$ for the variables $X_{2}^{2}, X_{3}^{2}$ ) without any specific intention. This latter step (the assignment of the proportions) is of crucial interest for the purposes of policy indications.

\subsection{The Efficiency Measurement: Two Approaches}

According to the relational approach (Kao, 2009a) once solved the model 1 above the efficiency of the system $\left(\mathrm{E}_{\mathrm{sys}}\right)$ is given by :

$$
E_{\text {sys }}=\frac{v_{1}^{*} Y_{3}+v_{2}^{*} Y_{4}+v_{3}^{*} Y_{5}}{u_{1}^{*} X_{1}+u_{2}^{*} X_{2}+u_{3}^{*} X_{3}+u_{4}^{*} X_{4}+u_{5}^{*} X_{5}}=v_{1}^{*} Y_{3}+v_{2}^{*} Y_{4}+v_{3}^{*} Y_{5}
$$

While the relative efficiency of the subprocesses will be produced with its constraints as follows::

$$
\begin{aligned}
E_{I} & =\frac{w_{1}^{*} Y_{1}+w_{2}^{*} Y_{2}}{u_{1}^{*} X_{1}^{1}+u_{2}^{*}\left(1-\alpha_{1}-\beta_{1}\right) X_{2}^{1}+u_{3}^{*}\left(1-\alpha_{2}-\beta_{2}\right) X_{3}^{1}} \\
E_{I I} & =\frac{v_{1}^{*} \delta_{1} Y_{3}^{(2)}+v_{2}^{*} \delta_{2} Y_{4}^{(2)}+v_{3}^{*} \delta_{3} Y_{5}^{(2)}}{u_{4} X_{4}+w_{1} \gamma_{1} Y_{1}^{(12)}+w_{2} \gamma_{2} Y_{2}^{(12)}+u_{2} \alpha_{1} X_{2}^{2}+u_{3} \alpha_{2} X_{3}^{2}} \\
E_{I I I} & =\frac{v_{1}^{*}\left(1-\delta_{1}\right) Y_{3}^{(3)}+v_{2}^{*}\left(1-\delta_{2}\right) Y_{4}^{(3)}+v_{3}^{*}\left(1-\delta_{3}\right) Y_{5}^{(3)}}{u_{5}^{*} X_{5}+u_{3}^{*} \beta_{2} X_{3}^{(3)}+u_{2}^{*} \beta_{1} X_{2}^{(3)}+w_{2}^{*} \pi_{2} Y_{2}^{(13)}+w_{1}^{*} \pi_{1} Y_{1}^{(13)}}
\end{aligned}
$$

Variables with an asterisk present the optimal value of the weights, once model 1 has been solved. As evident from formulas 2, 3, 4 the weights are the same for the same variable. Studies have proposed that, in the case of unstructured systems with multiple stages, remodeling the system as a structure in parallel with a series of subsystems using a dummy process is required (Kao, 2009(a)). In our opinion, the use of dummy processes remains an excellent solution for such systems. However, to estimate the relative efficiency of such systems and its sub-processes, we propose to build blocks by combining at least two sub-processes together without any dummy process. So, for the process represented in Figure 1, we build a block (Block 1) using subprocess 1 and 2 and we get a new system (see Figure 3). The initial system will then be transformed as follows: 


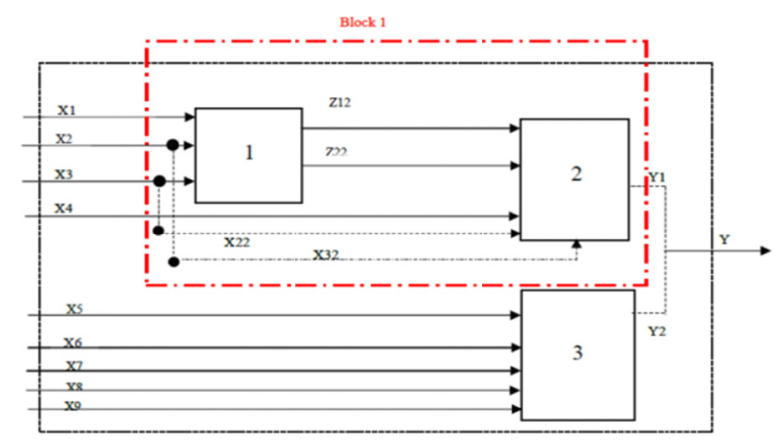

Figure 3. System with block

Block 1 is a well structured internal subsystem within the system, and in particular it is constituted as a two-part series system with shared inputs variables X22 and X32 respectively. (Kao, 2009(a)) (Castelli, et al., 2010) (Kao, 2014) (Kao, 2017). To build this block we assume that, it is possible to assign independent resources to the block by eliminating, partially or totally, the pre-existing links with the rest of the sub-processes, Figure 1 . We have eliminated both the inputs shared with stage 3 and the interconnections of intermediate variables with it, the gray and yellow lines Figure 1. We have assigned to all the shared variables both input and intermediate, between stage 3 and the remaining 1 and 2 the variables Y1, Y2, X2, X3 which are obtained by applying an attribution proportion to the corresponding system variables. System 3 has a new resource, the indlependent variables X6, $\mathrm{X} 7, \mathrm{X} 8$ and $\mathrm{X} 9$ obtained from the transformation on intermediate variables $\mathrm{Y} 1, \mathrm{Y} 2$ and on shared variables $\mathrm{X} 2$ and X3 along with the subprocess and its own variables (X5). In this case, the result of this strategy is the transformation of the original system into a system with a block 1 plus another subprocess which coincides with subprocess 3 with shared output Figure 3. This solution avoids entry of a dummy subprocess. This solution can be adopted for policy needs, but in our opinion, it also helps to improve both the interpretation and the measurement of the efficiency of the system and its sub-parts. In fact, once block 1 has been constructed, its efficiency can be measured as a series system by applying the basic relational NDEA model in series (Kao, 2009a). Thereby, measuring the efficiency of the third subprocess with an independent DEA model without shared outputs and also assigning it to this output variable. A proportion in the total output that will be partly attributed to block 1 and remaining to the third sub-process. While the inputs of sub-process 3 are in turn assigned in proportion to the total system resources. The importance is due to the measurement and the policy of this strategy. For example, when two subprocesses cannot be separated for technical or other reasons happening in treatment of hospital acute care. (Harris \& Autumn, 1977) Also, where it may not make sense to consider the nursing care activity separately from the medical ones and vice versa. While on the other hand, it might make sense to keep the rehabilitation service separate from the rest of the hospital services. A characteristic of our proposal is the possibility of considering the subsystem variables as independent. In other words, instead of considering beds as shared variables, as happens in the relational model, we can consider them as independent variables and can attribute them to the first subprocess (medical activity) and to the third subprocess (rehabilitation activity) according to managerial or the researcher preferences. The immediate implication, with regard to the relational model, is that the beds and technical-health personnel of the production process considered here, will receive different weights in the new transformed system, Figure 3. In this way, the efficiency measurement will affect Block 1 using the following relational NDEA model:

$$
\begin{gathered}
v_{1} \alpha Y 1 \\
\text { s.t. } u_{1} X 1+u_{2} X 2+u_{3} X 3+u_{4} X 4=\mathbf{1} \\
\left(w_{1} Z 12+w_{2} Z 22\right)-\left(u_{1} X 1+u_{2} \sigma_{1} X 2+u_{3} \sigma_{2} X 3+u_{4} X 4\right) \leq 0 \\
v \alpha Y 1-\left(w_{1} Z 12+w_{2} Z 22+u_{2}\left(1-\sigma_{1}\right) X 2+u_{3}\left(1-\sigma_{2}\right) X 3-u_{4} X_{4}\right) \leq 0 \\
v_{1} Y 1-\left(u_{1} X 1+u_{2} X 2+u_{3} X 3+u_{4} X 4\right) \leq 0
\end{gathered}
$$

(model 2 )

Where $\sigma_{1}, \sigma_{2}$ are the proportions of the variables $X_{2}$ and $X_{3}$ respectively assigned to the first subprocess and $\left(1-\sigma_{1}\right)$ and $\left(1-\sigma_{2}\right)$ the proportions of the same variables assigned to the second subprocess. $\alpha$ is the proportion of the variable $Y_{2}$ produced to the block 1, relatively to the whole output produced by the system $\mathrm{Y}=\mathrm{Y} 1+\mathrm{Y} 2$ (see Figure 2). To the model 2 we will obtain the following optimal weights: $v_{1}^{*}, u_{1}^{*}, u_{2}^{*}, u_{3}^{*}, u_{4}^{*}$. So, the efficiency of the block 1 will be: 


$$
E_{\text {Block } 1}=\frac{v_{1}^{*} \alpha Y 1}{\left(u_{1}^{*} X 1+u_{2}^{*} X 2+u_{3}^{*} X 3+u_{4}^{*} X 4\right)}=v_{1}^{*} \alpha Y 1
$$

Later we will solve the following standard DEA model for the remaining subprocess (here the subprocess 3 ):

$$
\begin{gathered}
v_{2} \beta Y 2 \\
\text { s.t. } \quad u_{5} X_{5}+u_{6} X_{6}+u_{7} X_{7}+u_{8} X_{8}+u_{9} X_{9}=1 \\
v_{2} \beta Y 2-\left(u_{5} X_{5}+u_{6} X_{6}+u_{7} X_{7}+u_{8} X_{8}+u_{9} X_{9}\right) \leq 0
\end{gathered}
$$

To the model 3 we will obtain the following optimal values: $v_{2}^{*}, u_{5}^{*}, u_{6}^{*}, u_{7}^{*}, u_{8}^{*}, u_{9}^{*}$. So, the efficiency of the third subprocess will be:

$$
E_{I I I}=\frac{v_{2}^{*} \beta Y 2}{u_{5}^{*} X_{5}+u_{6}^{*} X_{6}+u_{7}^{*} X_{7}+u_{8}^{*} X_{8}+u_{9}^{*} X_{9}}=v_{2}^{*} \beta Y 2
$$

The efficiency of the whole subprocess can be calculate using the weighted additive efficiency decomposition (Chen, et al., 2009b) as follow:

$$
E_{\text {sysblock }}=\tau_{1} E_{\text {block }}+\tau_{2} E_{I I I}
$$

Where $\tau_{1} \geq 0, \tau_{2} \geq 0$ with $\tau_{1}+\tau_{2}=1$, represent the importance of sub-process 3 and block 1 in measuring efficiency for the entire system. With this strategy, we treat the variables of subprocess 3 and those of the block as non-relational variables. In fact, as presented above, we will have different weights for each subprocess input variable. Thus, the variables X2 (of block 1) and X6 (of subprocess 3) represent the same resource (ie read in this example). The variable associated with it is assigned to block 1 and subprocess 3 as independent variables each with an own weight and in a given proportion. All data will be covered in the section dedicated to the application. In our opinion, this measurement strategy is very useful in technological, organizational and / or legislative constraints.

\section{The Application to the Hospitals}

The efficiency measurement in the health care/hospitals setting when we consider its internal structure is relatively recent.[i.e. (Chilingerian, et al., 2004); (Kawaguchi, et al., 2014); (Pinto, 2016)]. Chilingerian et al. 2004 consider a two stage process in measuring the physicians care and apply two separate DEA. The first stage has as inputs registered nurses, medical supplies, and capital and fixed costs. These inputs generate the outputs as patient days, quality of treatment, drugs dispensed, among others. These first stage outputs are the inputs of the second stage to generate as outputs research grants, quality patients, and quantity of individual trained, by speciality. Kawaguchi et al 2014 test the policy effects of the health reform in Japan on the hospital efficiency considering this latter as organizations with two internal heterogenous organizations. In particular the authors apply the dynamic-network data envelopment analysis. Pinto 2016 consider a two stage process in the hospitals acute care applying the network DEA approach to estimates the relative efficiency of it. In Pinto,2016 the second stage has an exogenous inputs conferring the non linearity to the model.In this paper we proposed in the subsection 3.2, according our opinion, a new approach in the case of a three stages process. In this section we apply it to the hospital acute care services adding a third process at the process in (Pinto, 2016). The variables used here are the same in (Pinto, 2016) (see Table 1)

Table 1. Hospital acute care's production process variables

\begin{tabular}{lcc}
\hline Relational model & Outputs & Relational variables \\
Inputs & Ordinary discharges & Surgical interventions \\
Physicians & Days-on hospitals \\
Ordinary beds & Sh-hospital discharges & Shared resources \\
Day-hospital beds & Surgical discharges & \\
Day-surgery beds & & \\
Nurses (second sub-process) & \\
Medical-technical staff (not included) & \\
Rehabilitative staff & & \\
\hline
\end{tabular}

The role of the some variables inside the relational model will depend to how the production process will be modeled. Here, the variables of the relational NDEA model in the case of hospitals acute care production process with three stages as in the Figure 1 above will be:

$\mathrm{X} 1, \mathrm{X} 2, \mathrm{X} 3, \mathrm{X} 4, \mathrm{X} 5=$ system resources: physicians, nurses, beds, rehabilitative staff, medical-technical-staff.

Y3, Y4, Y5= outputs system:ordinary discharges, day-hospital discharges, surgical discharges. 
$\mathrm{X}_{1}^{(1)}, \mathrm{X}_{2}{ }^{(1)}, \mathrm{X}_{3}{ }^{(1)}=$ resources of the I sub-process:physicians, beds, medical-technical-staff.

$\mathrm{Y}_{1}, \mathrm{Y}_{2}=$ outputs of the I sub- process :surgical interventions, days on hospitals.

$\mathrm{Y}_{1}{ }^{(12)}, \mathrm{Y}_{2}{ }^{(12)}=$ relational resources between the I and the II sub-process:surgical interventions, days on hospitals.

$\mathrm{Y}_{1}{ }^{(13)}, \mathrm{Y}_{2}{ }^{(13)}=$ relational resources between the I and the III sub-process:surgical interventions, days on hospitals.

$\mathrm{X}_{2}{ }^{(2)}, \mathrm{X}_{3}{ }^{(2)}=$ shared inputs resources between the I and the II sub-process (beds, medical-technical-staff).

$\mathrm{X} 4=$ exogenous resources of the II sub-process:nurses.

$\mathrm{Y}_{3}^{(2)}, \mathrm{Y}_{4}^{(2)}, \mathrm{Y}_{5}^{(2)}=$ outputs of the II subprocesses: ordinary discharges, day-hospital discharges,surgical discharges.

$\mathrm{X} 5=$ resources of the III sub-process: rehabilitative staff.

$\mathrm{X}_{2}{ }^{(3)}, \mathrm{X}_{3}{ }^{(3)}$,= shared resources between I and III sub-process: beds, medical-technical-staff.

$\mathrm{Y}_{1}{ }^{(13)}, \mathrm{Y}_{2}{ }^{(13)}=$ shared outputs resources between I and III subprocesses: surgical interventions, patients days. $\mathrm{Y}_{3}{ }^{(3)}, \mathrm{Y}_{4}{ }^{(3)}, \mathrm{Y}_{5}{ }^{(3)}=$ outputs of the third sub-process:ordinary discharges, day-hospital discharges, surgical discharges,

$\alpha_{1}, \alpha_{2}=$ proportion of the shared inputs variables $\mathrm{X}_{2}^{(2)}, \mathrm{X}_{3}^{(2)}$.

$\beta_{1}, \beta_{2}=$ proportion of the shared inputs variables $\mathrm{X}_{2}{ }^{(3)}, \mathrm{X}_{3}{ }^{(3)}$.

$\gamma_{1}, \gamma_{2}=$ proportion of the shared intermediate variables $\mathrm{Y}_{1}^{(12)}, \mathrm{Y}_{2}^{(12)}$ and,

$\pi_{1}, \pi_{2}=$ proportion of shared intermediate variables between the I and III subprocesses $\mathrm{Y}_{1}^{(13)}, \mathrm{Y}_{2}^{(13)}$.

$\delta_{1}, \delta_{2} . \delta_{3}=$ is the proportion of the shared outputs variables $\mathrm{Y}_{3}^{(2)}, \mathrm{Y}_{4}^{(2)}, \mathrm{Y}_{5}^{(2)}$ and $(1-\delta)$ is the proportion of, $\mathrm{Y}_{3}{ }^{(3)}$, $\mathrm{Y}_{4}{ }^{(3)}, \mathrm{Y}_{5}{ }^{(3)}$.

As noted, differently to (Pinto, 2016) here we added a third subprocesses to modeling the rehabilitative activity using as dedicated variable the rehabilitative staff. (see Table 1). This latter variable characterizing the third subprocesses in the model (lacking in (Pinto, 2016)). Solving the model 1 and applying the formulas 1,2,3,4 using data on these variables we will have the following optimal weights (additional file1 .xlsx ) and the following relative efficiencies (see Table 2 below for its descriptive statistics).

Table 2. Descriptive statistics of NDEA relational efficiency and its subprocess

\begin{tabular}{|c|c|c|c|c|c|}
\hline Statics & $\begin{array}{c}\text { Relational efficiency } \\
\text { (formula 1) }\end{array}$ & $\begin{array}{l}\text { Esub1 (formula } \\
\text { 2) }\end{array}$ & $\begin{array}{c}\text { Esub2 } \\
\text { (formula 3) }\end{array}$ & Esub3 (formula 4) & $\begin{array}{c}\text { Eaggr } \\
(\text { Esub1 } * \text { Esub2 } 2 \text { Esub3) }\end{array}$ \\
\hline nbr.val & 554,0000 & 552,0000 & 554,0000 & 552,0000 & 552,0000 \\
\hline nbr.null & 2,0000 & 0,0000 & 2,0000 & 0,0000 & 0,0000 \\
\hline nbr.na & 0,0000 & 2,0000 & 0,0000 & 2,0000 & 2,0000 \\
\hline $\min$ & 0,0000 & 0,0047 & 0,0000 & 0,0485 & 0,0002 \\
\hline $\max$ & 0,9996 & 1,0000 & 1,0000 & 1,0000 & 1,0000 \\
\hline range & 0,9996 & 0,9953 & 1,0000 & 0,9515 & 0,9998 \\
\hline sum & 342,7996 & 366,2543 & 372,5293 & 335,0617 & 170,6967 \\
\hline median & 0,6253 & 0,6794 & 0,6741 & 0,6047 & 0,2740 \\
\hline mean & 0,6188 & 0,6635 & 0,6724 & 0,6070 & 0,3092 \\
\hline SE.mean & 0,0078 & 0,0067 & 0,0080 & 0,0079 & 0,0085 \\
\hline CI.mean.0.9 & 0,0154 & 0,0131 & 0,0158 & 0,0155 & 0,0167 \\
\hline \multicolumn{6}{|l|}{5} \\
\hline var & 0,0341 & 0,0244 & 0,0358 & 0,0344 & 0,0397 \\
\hline std.dev & 0,1846 & 0,1563 & 0,1893 & 0,1856 & 0,1992 \\
\hline coef.var & 0,2983 & 0,2356 & 0,2815 & 0,3057 & 0,6441 \\
\hline skewness & $-0,4455$ & $-0,5014$ & $-0,5975$ & $-0,1548$ & 1,1992 \\
\hline skew.2SE & $-2,1463$ & $-2,4112$ & $-2,8784$ & $-0,7446$ & 5,7670 \\
\hline kurtosis & 0,7708 & 1,1507 & 0,8864 & 0,5645 & 1,6994 \\
\hline kurt.2SE & 1,8599 & 2,7718 & 2,1389 & 1,3597 & 4,0933 \\
\hline normtest.W & 0,9735 & 0,9744 & 0,9636 & 0,9766 & 0,9155 \\
\hline normtest.p & 0,0000 & 0,0000 & 0,0000 & 0,0000 & 0,0000 \\
\hline
\end{tabular}

Legend: ndr.var:number of observations; nrr.null: number of null values; nbr.na:number of missing value; min;max;range;sum: sum of all non-missing values ;media;mean;SE.mean:standard error on the mean ; CI.mean.:the confidence interval of the mean at the p level; var:; stad.var;coef.var:variation coefficient defined as the standard deviation divided by the mean ;skewness:skewness coefficient ;skew.2SE:its significant criterium ( if skew.2SE > 1, then skewness is significantly different than zero), ;kurtosis:kurtosis coefficient ;kurt.2SE:its significant criterium;normtest.W:statistic of a Shapiro-Wilk test of normality ;normtest.p:its associated probability. 
Instead, adopting the other model above (model 2) we will have the following variables:

$\mathrm{X} 1, \mathrm{X} 2, \mathrm{X} 3, \mathrm{X} 4=$ block 1 resources: physicians, beds, medical-technical-staff, nurses.

$\mathrm{Y} 1=$ block 1 outputs:ordinary discharges, day-hospital discharges, surgical discharges.

$\mathrm{Z} 11, \mathrm{Z} 22$ = intermediate variables block 1: surgical interventions, days on hospitals.

X22, X32= block 1shared resources:beds,, medical-technical-staff.

Solving the model 2 and 3 and applying the formulas 5, 6, 7 we will have the following efficiencies scores (see Table 2) and optimal weights (additional file 2 and 3. xlsx):

Table 3. Descriptive statistics of system efficiency with block and block efficiency

\begin{tabular}{|c|c|c|c|c|c|}
\hline Statistics & $\begin{array}{c}\text { Esysblock1 (formula } \\
7 \text { ) }\end{array}$ & $\begin{array}{c}\text { Esysblock1a (formuma } \\
\text { 7) }\end{array}$ & $\begin{array}{l}\text { Esysblock1b (formula } \\
\text { 7) }\end{array}$ & $\begin{array}{l}\text { Eblock1 (formula } \\
\text { 5) }\end{array}$ & $\begin{array}{c}\text { effstage } 3 \text { (formula } \\
6 \text { ) }\end{array}$ \\
\hline nbr.val & 554,0000 & 554,0000 & 554,0000 & 554,00010 & 554,0000 \\
\hline nbr.null & 2,0000 & 2,0000 & 2,0000 & 2,0000 & 2,0000 \\
\hline nbr.na & 0,0000 & 0,0000 & 0,0000 & 0,0000 & 0,0000 \\
\hline $\min$ & 0,0000 & 0,0000 & 0,0000 & 0,0000 & 0,0000 \\
\hline $\max$ & 1,0000 & 1,0000 & 1,0000 & 1,0000 & 1,0000 \\
\hline range & 1,0000 & 1,0000 & 1,0000 & 1,0000 & 1,0000 \\
\hline sum & 371,5069 & 372,2177 & 369,3743 & 368,6634 & 375,7720 \\
\hline median & 0,6757 & 0,6749 & 0,6720 & 0,6700 & 0,6821 \\
\hline mean & 0,6706 & 0,6719 & 0,6667 & 0,6655 & 0,6783 \\
\hline SE.mean & 0,0085 & 0,0085 & 0,0085 & 0,0085 & 0,0088 \\
\hline $\begin{array}{l}\text { CI.mean.0.9 } \\
5\end{array}$ & 0,0167 & 0,0167 & 0,0167 & 0,0167 & 0,0174 \\
\hline var & 0,0399 & 0,0401 & 0,0399 & 0,0401 & 0,0433 \\
\hline std.dev & 0,1997 & 0,2003 & 0,1997 & 0,2004 & 0,2081 \\
\hline coef.var & 0,2978 & 0,2982 & 0,2996 & 0,3011 & 0,3068 \\
\hline skewness & $-0,5775$ & $-0,5760$ & $-0,5532$ & $-0,5336$ & $-0,5319$ \\
\hline skew.2SE & $-2,7823$ & $-2,7749$ & $-2,6653$ & $-2,5704$ & $-2,5626$ \\
\hline kurtosis & 0,6047 & 0,5757 & 0,6106 & 0,5927 & 0,3847 \\
\hline kurt.2SE & 1,4593 & 1,3893 & 1,4733 & 1,4302 & 0,9284 \\
\hline normtest.W & 0,9642 & 0,9644 & 0,9648 & 0,9648 & 0,9602 \\
\hline normtest.p & 0,0000 & 0,0000 & 0,0000 & 0,0000 & 0,0000 \\
\hline
\end{tabular}

In the Table 2 the additive efficiency aggregation (formula 7) it has been calculate using different values of $\tau$. In particular: Esysblock1 consider $\tau_{1}=0.6, \tau_{2}=0.4$, Esysblock1a $\tau_{1}=0.5, \tau_{2}=0.5$ and Esysblock $1 \mathrm{~b} \tau_{1}=$ $0.9, \tau_{2}=0.1$. To compare the efficiencies of the two models (model 1 relational NDEA, and model 2 network system with block) we plot the empirical cumulative distribution functions (see Figure 4), also.

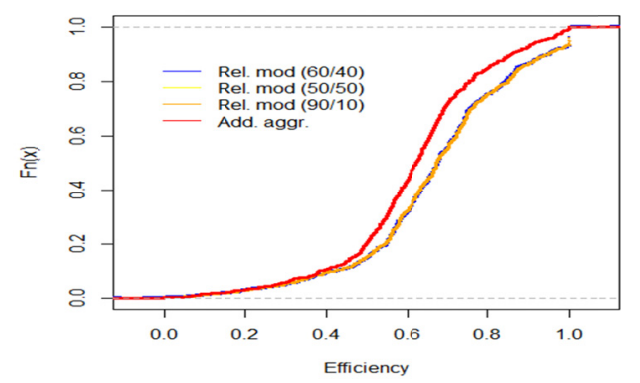

Figure 4. Empirical cumulative distribution of efficiency scores model 1 and 2

At the same way we compare the efficiencies of the subprocess 3 (see Figure 5). 


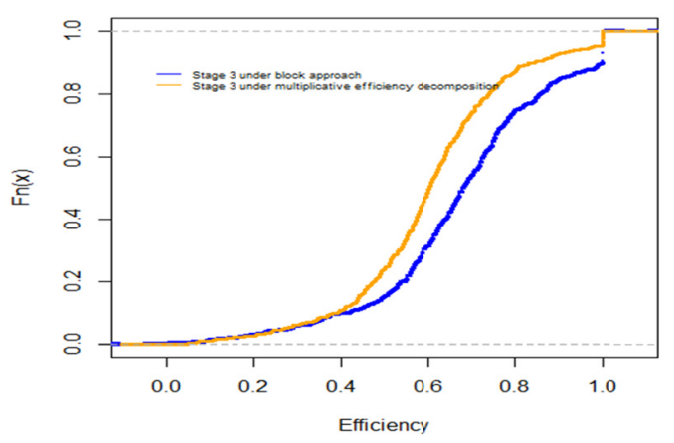

Figure 5. Empirical cumulative distribution of model 1 and 2 of third subprocess efficiency scores

The blue curve is the cumulative empirical distribution function of the third stage efficiency scores calculated with an independent standard DEA model in line with the block approach (formula 6) proposed here while the arange curve is the cumulative empirical distribution function of the relative efficiency score always of the sub process 3 but calculated using the multiplicative decomposition formula applied to the relational NDEA model (formula 4). The two curves are very different. This is the effect of the different optimall weights calculated for the same variable in the two approaches listed above. In other words, if we consider the bed variable, this variable receives the same weight when it is in the relational model. In the case of the block approach the subprocess 3 becomes independent of block 1 and two different weights are assigned. It is a variable of block 1 and other when it is an input variable of subprocess 3 . Since, the weight variables have evident policy value, the two approaches, relational and block, lead to different policy considerations both for the whole system and for its parts (Smith, et al., 2005). The descriptive statistics of the efficiency measurements for sub-process 3 under the two approaches are reported in Table 4.

Table 4. Efficiency scores of the third subprocess under model1 and model 2

\begin{tabular}{lcc}
\hline Statistics & effstage3 (formula & Esub3 (formula \\
& 6 ) & 4 ) \\
\hline nbr.val & 554,0000 & 552,0000 \\
nbr.null & 2,0000 & 0,0000 \\
nbr.na & 0,0000 & 2,0000 \\
min & 0,0000 & 0,0485 \\
max & 1,0000 & 1,0000 \\
range & 1,0000 & 0,9515 \\
sum & 375,7720 & 335,0617 \\
median & 0,6821 & 0,6047 \\
mean & 0,6783 & 0,6070 \\
SE.mean & 0,0088 & 0,0079 \\
CI.mean.0.9 & 0,0174 & 0,0155 \\
5 & & \\
var & 0,0433 & 0,0344 \\
std.dev & 0,2081 & 0,1856 \\
coef.var & 0,3068 & 0,3057 \\
skewness & $-0,5319$ & $-0,1548$ \\
skew.2SE & $-2,5626$ & $-0,7446$ \\
kurtosis & 0,3847 & 0,5645 \\
kurt.2SE & 0,9284 & 1,3597 \\
normtest.W & 0,9602 & 0,9766 \\
normtest.p & 0,0000 & 0,0000 \\
\hline
\end{tabular}

Although in Table 4, there are small differences in the average value of the efficiency scores, their distribution is very different as we have seen in Figure 4. In fact, the cumulative empirical distribution function of the efficiency score obtained under the multiplication decomposition formula is very concave than observed for the efficiency scores calculated with the block approach (subsection 3.2). The DEA efficiency scores (1) are shown in Table 5 below. 
Table 5. DEA efficiency score: the black box performance measurement

\begin{tabular}{lll}
\hline Statistics & DEA-D & DEA-ND \\
\hline nbr.val & 554 & 554 \\
nbr.null & 2 & 2 \\
nbr.na & 0 & 0 \\
min & 0 & 0 \\
max & 1 & 1 \\
range & 1 & 1 \\
sum & 464,7384 & 469,1283 \\
median & 0,857498 & 0,865461 \\
mean & 0,838878 & 0,846802 \\
SE.mean & 0,006275 & 0,006296 \\
CI.mean.0.95 & 0,012325 & 0,012367 \\
var & 0,021812 & 0,02196 \\
std.dev & 0,14769 & 0,14819 \\
coef.var & 0,176057 & 0,174999 \\
skewness & $-1,69626$ & $-1,7123$ \\
skew.2SE & $-8,17169$ & $-8,24899$ \\
kurtosis & 5,692925 & 5,578384 \\
kurt.2SE & 13,73721 & 13,46082 \\
normtest.W & 0,864577 & 0,855679 \\
normtest.p & $1,77 \mathrm{E}-21$ & $4,02 \mathrm{E}-22$ \\
\hline
\end{tabular}

The column DEA-D refer to the DEA score efficiency with all discretionary inputs, while the column DEA-ND refer DEA efficiency scores in the case of not discretionary inputs. In this case we posed the variables nurse as not discretionary as example. We can see the effect of the non discretionary asisumption, in terms of performance management, on the values of the descriptive statistic in the Table 5 above and in the histogram and empirical cumulative distribution function of the DEA efficiency score in the Figure 6 below.
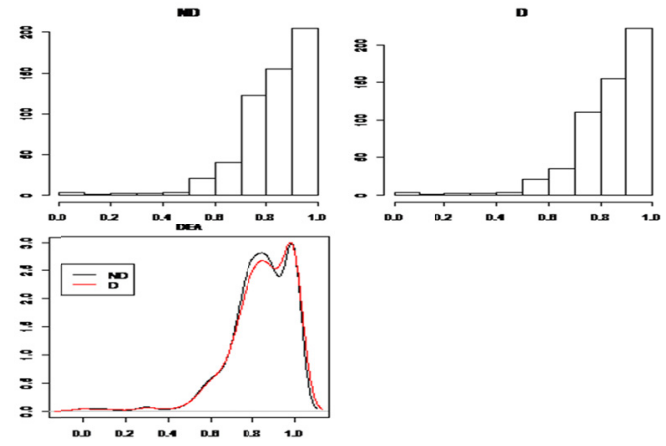

Figure 6. Empirical cumulative distributions DEA scores and histograms

\section{Discussion and Conclusions}

The economic modeling of a production process is very often conducted, using the production function approach to represent the maximum level of output attainable for each level of resources. Within an econometric model, it is possible to estimate the production function and measure the efficiency similar to SFA [ (Battese, et al., 1977)]. In other cases, the measurement of the efficiency of a production process is carried out using a mathematical model as happens in the case of DEA (Cooper, et al., 2007). In these two cases, however, it is impossible to think of the production process as a system made up of several connected parts. Some authors, introduced the idea of modeling a production process as a system of interconnected parts [ (Fa"re, et al., 1996a); (Färe, et al., 1996b)] and since then great progress has been made in this direction [ (Kao, 2014); (Kao, 2009(a)); (Kao, 2017)]. Furthermore, two basic models: one with a series structure and the other with a parallel structure had developed a network model within the DEA method to measure its relative efficiency (Kao, 2009(a)). Here, we have modeled a production process as a system of three interconnected sub-processes or 
stages, Figure 1. For demonstration purposes, we applied this model to the case of the hospital acute care production process by exploiting the conceptualization (Pinto, 2016). The process in (Pinto, 2016) was enriched by connecting the rehabilitation activity along with medical care and assistance with activities / subprocesses, in a treatment process for hospital acute care. We later developed a relational NDEA model to measure its relative efficiency(model 1) and calculate its subprocess efficiency using multiplicative decomposition formula (Kao, 2009(a)). As innovative way to measure its relative efficiency we proposed as an alternative solution for construction of an internal block of two subprocesses. In this latter case we not apply the relational NDEA approach directly to the whole process to estimates its relative efficiency. Once the relative efficiency of the block 1 in Figure 3 was calculated with a relational NDEA model, we applied the multiplicative decomposition formula for the block to calculate the relative efficiency of its subprocesses. To calculate the efficiency of the entire process, we have adopted the additive decomposition formula with the efficiency of the block and the efficiency of the remaining sub-process (Chen, et al., 2009b). In other words, our solution, one obtained an internal block, combines relational NDEA with DEA to measure the relative efficiency of network systems with more than two subprocesses. However, once the block has been constructed, a specific weight can be assigned to it, reflecting its importance within the measurement. Our approach, according our opinion, is useful for specific policy indications. Obviously, blocking can be differently constructed based on the requirements of the policy objectives, and under the constraint of a network model of two sub-processes resulting from the basic ones in series and in parallel (Kao, 2009(a)). The relational NDEA 1 proposed model, is characterized by intermediate flows, shared variables and exogenous variables (Castelli, et al., 2010) and the same variable receive the same weight- A variant not considered here, is that which uses exogenous variables to give the model a multi-stage nature (Kao, 2017). The difference between the direct application to the relational NDEA model (model 1) and our strategy, is that the former requires the use of the same weights for the same variables regardless of whether they belong to one or the other subprocesses (Kao, 2009a). In our approach to the same variable they can be assigned to different weight depending on whether it belongs to the residual block or sub-process, and avoid to insert a dummy process (Kao, 2009a). In this way, generating different optimal values in the weights and therefore different policy considerations (Smith, et al., 2005). We would like to conclude, that the study represents an innovative work for introducing the block strategy in the measurement of relative efficiency of systems with more than two subprocesses. Moreover, it is new in view of the measurement of relative hospital efficiency with NDEA models. In addition, it can be applied for different technological, legislative, etc, constraints where two subprocesses in a network system are joint.

\section{References}

Aigner, D. J., Lovell, C. A. K., \& Schmidt, P. (1977). Formulation and estimation of stochastic frontier production functions. Journal of Econometrics, 6, 21-37. https://doi.org/10.1016/0304-4076(77)90052-5

Battese, G. E., \& Corra, G. S. (1977). Estimation of a production frontier model: with application to the pastoral zone of eastern australia. Australian Journal of Agricultural and Resource Economics, 21(3). https://doi.org/10.1111/j.1467-8489.1977.tb00204.x

Castelli, L., Pesenti, R., \& Ukovich, W. (2004). DEA-like models for the efficiency evaluation of hierarchically structured units. European Journal of Operational Research, 154(2), 465-476. https://doi.org/10.1016/S0377-2217(03)00182-6

Castelli, L., Pesenti, R., \& Ukovich, W. (2010). A classification of DEA models when the internal structure of the Decision Making Units is considered. Annals of Operations Research, 173, 207-235. https://doi.org/10.1007/s10479-008-0414-2

Charnes, A., Cooper, W. W., \& Rhodes, E. (1978). Measuring the efficiency of decision making units. European Journal of Operational Research, 2, 429-444. https://doi.org/10.1016/0377-2217(78)90138-8

Chen, Y. et al. (2009). Additive efficiency decomposition in two-stage DEA. European Journal of Operational Research, 196, 1170-1176. https://doi.org/10.1016/j.ejor.2008.05.011

Chen, Y. et al. (2010). DEA model with shared resources and efficiency decomposition. European Journal of Operational Research, 207, 339-349. https://doi.org/10.1016/j.ejor.2010.03.031

Chen, Y., \& Zhu, J. (2004). Measuring information technology's indirect impact on firm performance. Information Technology \& Management Journal, 5(1-2), 9-22. https://doi.org/10.1023/B:ITEM.0000008075.43543.97 
Chilingerian, J., \& Sherman, H. D. (2004). Health care applications: From Hospitals to Physician, from productive efficiency to quality frontiers. In L. M. Seiford, \& J. Zhu W. W. Cooper (Eds.), Handbook on data envelopment analysis. Boston: Springer.

Cook, W. D., \& Seiford, L. M. (2009). Data envelopment analysis (DEA) - thirty years on. European Journal of Operational Research, 192, 1-17. https://doi.org/10.1016/j.ejor.2008.01.032

Cooper, W. W., Seiford, L. M., \& Tone, K. (2007). Data Envelopment Analysis. A Comprehensive Text with Models, Application, References and DEA-Solver Software. New York: Springer.

Cooper, W. W., Seiford, L. M., \& Tone, K. Z. J. (2007). Some models and measures for evaluating performances with DEA:past accomplishments and future prospects. Journal of Productivity Analysis, 28, 151-163. https://doi.org/10.1007/s11123-007-0056-4

Daraio, C., \& Simar, L. (2008). Advanced Robust and Nonparametric Methods in Efficiency Analysis. Methodology and Applications. s.l.: Springer.

Deprins, D., Simar, L., \& Tulkens, H. (1984). Measuring Labor-Efficiency in Post Office. [aut. libro] M in Marchand, P. Pestieu e H. Tulkens. The performance of Public Enterprises: Concepts and Measurement, 243-267.

Färe, R., \& Grosskopf, S. (1996a). Intertemporal production frontiers: with dynamic DEA. Boston: Kluwer. https://doi.org/10.1007/978-94-009-1816-0

Färe, R., \& Grosskopf, S. (1996b). Productivity and intermediate products:A frontier approach. Economics Letters, 50, 65-70. https://doi.org/10.1016/0165-1765(95)00729-6

Fa“re, R., \& Whittaker, G. (1995). An intermediate input model of dairy production using complex survey data. $J$ Agric Econ, 46, 201-213. https://doi.org/10.1111/j.1477-9552.1995.tb00766.x

Greene, W.H. 1980. Maximum likelihood estimation of econometric frontier functions. J. Econometrics. 1980 , Vol. 13:, p. 27-56. https://doi.org/10.1016/0304-4076(80)90041-X

Harris, J. E. (1977). The Internal Organization of Hospitals: Some Economic Implications. The Bell Journal of Economics, 8(2), 467-482. https://doi.org/10.2307/3003297

Hiller, F. S., \& Lieberman, G. J. (2001). Introduction to Operation Research. McGraw-Hill Series in Industrial Engineering and Management Science.

Kao, C. (2009a). Efficiency decomposition in network data envelopment analysis: A relational model. European Journal of Operational Research, 192, 949-962.

Kao, C. (2014). Efficiency decomposition for general multi-stage systems in data envelopment analysis. European Journal of Operational Research, 232, 117-124. https://doi.org/10.1016/j.ejor.2013.07.012

Kao, C. (2014). Network data envelopment analysis: A review. European Journal of Operational Research, 239(1), 1-16.

Kao, C. (2017). Network Data Envelopment Analysis. Foundation and Extension. s.1.: Springer.

Kao, C., \& Hwang, S. N. (2008). Efficiency decomposition in two-stage data envelopment analysis: An application to non-life insurance companies in Taiwan. European Journal of Operational Research, 185, 418-429. https://doi.org/10.1016/j.ejor.2006.11.041

Kawaguchi, H., Tone, K., \& Tsutsui, M. (2014). Estimation of the efficiency of Japanese hospitals using dynamic and network Data envelopment Analysis model. Health Care Management Sciences, 17, 101-112. https://doi.org/10.1007/s10729-013-9248-9

Lewis, H. F., \& Sexton, T. R. (2004). Network DEA: efficiency analysis of organizations with complex internal structure. Computers \& Operations Research, 31(9), 1365-1410. https://doi.org/10.1016/S03050548(03)00095-9

Liang, L. et al. (2006). DEA models for supply chain efficiency evaluation. Annals of Operations Research, 145(1), 35-49. https://doi.org/10.1007/s10479-006-0026-7

Liang, L., Cook, W. D., \& Zhu, J. (2008). DEA models for two-stage processes: Game approach and efficiency decomposition. Naval Research Logistics, 55, 643-653. https://doi.org/10.1002/nav.20308

Liu, J. S. et al. (2013). A survey of DEA applications. Omega, 41, 893-902. https://doi.org/10.1016/j.omega.2012.11.004 
Meeusen, W., \& Van den Broeck, J. (1977). Efficiency Estimation from Cobb-Douglas Production Functions with Composed Error. International Economic Review, 18(2), 435-44. https://doi.org/10.2307/2525757

Olesen, O. B., \& Petersen, N. C. (2016). Stochastic Data Envelopment Analysis-A review. European Journal of Operational Research, 251(1), 2-21. https://doi.org/10.1016/j.ejor.2015.07.058

Ozcan, Y. A. (2008). Health Care Benchmarking and Performance Evaluation. An Assessment using Data Envelopment Analysis (DEA). New York: Springer. https://doi.org/10.1007/978-0-387-75448-2

Pinto, C. (2016). The Acute Care Services Production Process's Efficiency: A DEA Network Model for the Italian Hospitals. Athens Journal of Health, 3(1).

Pinto, C. (2018). Performances management when modelling internal structure. w.p. MPRA. Retrieved from https://mpra.ub.uni-muenchen.de/87923/

Premachandra, I. M. et al. (2012). Best-performing US mutual fund families from 1993 to 2008: Evidence from a novel two-stage DEA model for efficiency decomposition. Journal of Banking and Finance, 36(12), 3302-3317. https://doi.org/10.1016/j.jbankfin.2012.07.018

Robinson, S. (2008). Conceptual modelling for simulation part I: definition and requirements. Journal of the Operational Research Society, 59(3), 278-290. https://doi.org/10.1057/palgrave.jors.2602368

Seiford, L. M. (1996). Data envelopment analysis:the evolution of the state of the art (1978-1995). Journal Of Productivity Analysis, 7, 99-137. https://doi.org/10.1007/BF00157037

Sexton, T., \& Lewis, H. (2003). Two-stage DEA: An application to Major League Baseball. Journal of Productivity Analysis, 19(2), 227-249. https://doi.org/10.1023/A:1022861618317

Smith, P. C., \& Street, A. (2005). Measuring the Efficiency of Public Services:The Limits of Analysis. Journal of the Royal Statistical Society, 168(2), 401-417. https://doi.org/10.1111/j.1467-985X.2005.00355.x

Zhu, J. (2009). Quantitative Models For Performance Evaluation and Benchmarking. Data Envelopment Analysis with Spreadsheets. New York: Springer. https://doi.org/10.1007/978-0-387-85982-8

\section{Notes}

Note 1. The approach refer to the relative efficiency. In other words the efficiency of a production process is measured relatively to a benchmark

Note 2. This is more an advances in the DEA modeling than a weakness.

Note 3. Here we no consider this variable.

Note 4. For the production of others services or goods we will have different activities with differents inputs and outputs. We can think for example to apply the model developed in the paper to modeling a simplified version of the wine production with the following three stages: stage 1-> harvesting, crushing, pressing, stage 2 $>$ fermentation, stage 3 (for some type of wine)- $>$ deraspaturing, with the pressed material as intermediate variable and as final outputs three types of wines: rosè (without deraspaturaing) + red and blank (with deraspaturaing). A proportion of the pressed material go to the third stage to be deraspaturaing and became red and blank wine.

Note 5. This mean that is not considered the deliver of rehabilitative services for outpatients. In terms of our model this mean add an external inputs to the rehabilitative stage (stage 3 in the Figure 1).

Note 6 . In the model with administrative staff, here do not treated, the assistance activity and the rehabilitative activities will finish when inpatient will be transformed in discharged through the delivery of administrative services by the administrative staff. This precisation is relevant to the functioning of the process but irrelevant for the relative efficiency measurement.

Note 7. In his book (Kao, 2017) shoe how in the network DEA model the efficiency measurement can be conduct using three model as in the counter part DEA model: 1) multiplier form ,2) envelopment form and 3) slacke-based form.

\section{Copyrights}

Copyright for this article is retained by the author(s), with first publication rights granted to the journal.

This is an open-access article distributed under the terms and conditions of the Creative Commons Attribution license (http://creativecommons.org/licenses/by/4.0/). 